\title{
Silent Myocardial Perfusion Abnormalities Detected by Stress Cardiovascular Magnetic Resonance in Antiphospholipid Syndrome: A Case-Control Study
}

\author{
Sophie I. Mavrogeni ${ }^{1}$, George Markousis-Mavrogenis ${ }^{1}$, Olga Karapanagiotou ${ }^{1}$, \\ Konstantinos Toutouzas ${ }^{2}$, Panagiotis Argyriou ${ }^{3}$, Stella Velitsista ${ }^{3}$, George Kanoupakis ${ }^{3}$, \\ Dimitrios Apostolou ${ }^{3}$, David Hautemann ${ }^{4}$, Petros P. Sfikakis ${ }^{5}$ and Maria G. Tektonidou ${ }^{5, *}$ \\ 1 Onassis Cardiac Surgery Center, 17674 Athens, Greece \\ 2 First Cardiology Department, Medical School, National and Kapodistrian University of Athens, \\ 11527 Athens, Greece \\ 3 Radiology Department, Mediterraneo Hospital, 16675 Athens, Greece \\ 4 Leiden University Medical Center, 2333 ZA Leiden, The Netherlands \\ 5 First Department of Propaedeutic Internal Medicine, Joint Rheumatology Program, Medical School, \\ National and Kapodistrian University of Athens, 11527 Athens, Greece \\ * Correspondence: mtektonidou@gmail.com
}

Received: 19 June 2019; Accepted: 20 July 2019; Published: 23 July 2019

\begin{abstract}
Objective: To examine the prevalence of silent myocardial ischemia and fibrosis in antiphospholipid syndrome (APS), using stress cardiovascular magnetic resonance (CMR). Methods: Forty-four consecutive APS patients without prior cardiac disease (22 primary APS, 22 systemic lupus erythematosus (SLE)/APS, mean age 44 (12.9) years, 64\% women) and 44 age/gender-matched controls were evaluated using CMR at $1.5 \mathrm{~T}$. Steady-state free precession imaging for function assessment and adenosine stress-CMR for perfusion-fibrosis evaluation were employed. The myocardial perfusion reserve index (MPRI), and myocardial fibrosis expressed as late gadolinium enhancement (LGE), were evaluated. Coronary angiography was indicated in patients with LGE. Associations with APS characteristics, classic cardiovascular disease (CVD) risk factors, high-sensitivity CRP (hs-CRP) and high-sensitivity Troponin (hs-TnT) levels were tested. All patients were followed up for 12 months. Results: Median MPRI was significantly lower in APS patients versus controls [1.5 (0.9-1.9) vs. 2.7 $(2.2-3.2), p<0.001]$, independently of any LGE presence. LGE was detected in 16 (36.3\%) patients versus none of controls $(p<0.001) ; 12 / 16$ were subsequently examined with coronary angiography and only two of them had coronary artery lesions. In multivariable analysis, none of the APS-related and classic CVD risk factors, or hs-CRP and hs-TnT covariates, were significant predictors of abnormal MPRI or LGE. At the twelve month follow-up, three (6.8\%) patients experienced coronary artery disease, notably those with the lowest MPRI values. Conclusions: Abnormal MPRI and LGE are common in asymptomatic APS patients, independently so of any APS-related and classic CVD risk factors, or coronary angiography findings in cases with LGE. Stress-CMR is a valuable tool to detect silent myocardial ischemia and fibrosis in APS.
\end{abstract}

Keywords: cardiovascular disease; autoimmune rheumatic diseases; antiphospholipid syndrome; cardiovascular magnetic resonance; ischemic cardiac disease; myocardial ischemia; myocardial fibrosis; late gadolinium enhancement

\section{Introduction}

Antiphospholipid syndrome (APS) is a rare systemic autoimmune disorder characterized by vascular thrombosis of large, medium or small vessels, pregnancy morbidity and persistently positive 
antiphospholipid antibodies (aPL), including lupus anticoagulant, anticardiolipin antibodies and/or anti- $\beta 2$-glycoprotein I antibodies [1]. These antibodies may be detected individually or in combinations of two or three (double- or triple-positive aPL, respectively). APS may occur in its primary form (primary APS, PAPS) or in correlation with other autoimmune diseases, mainly systemic lupus erythematosus (SLE/APS) [2].

Heart involvement is one of the major complications in APS, including valve and ischemic heart disease [3-5]. In a multi-center European cohort of 1000 patients with APS, heart valve disease-mostly valve thickening and/or vegetation-was observed in 30\% of patients, while ischemic heart disease manifesting mainly as myocardial infarction (MI), was demonstrated in $5.5 \%$ of patients [2]. MI was the most common cause of death after bacterial infections among patients with APS, referring to $19 \%$ of deaths in a 5-year follow-up period [2]. Other types of heart disease have also been reported in APS, including coronary vasospasm, known as variant (Prinzmetal's) angina and syndrome X [6,7], myocardial ischemia associated with thrombotic cardiac microvasculopathy [8], and endomyocardial fibrosis due to coronary microcirculation defects [9].

Cardiovascular magnetic resonance imaging (CMR) is a non-invasive, non-ionizing radiation imaging modality that can assess cardiac geometry and function, myocardial perfusion and fibrosis $[10,11]$. Only one previous study used CMR in APS that showed a significantly higher prevalence of occult myocardial fibrosis associated with microvascular disease, expressed as late gadolinium enhancement (LGE), in 27 patients with APS compared to 81 healthy controls [12]. Using exercise or pharmacological stress with adenosine (stress CMR), a three- to four-fold increase in myocardial blood flow (MBF) can occur in healthy individuals. The ratio of maximum stress MBF after adenosine use to baseline rest is defined as a myocardial perfusion reserve index (MPRI) (10). The MPRI indicates the functional severity of a coronary lesion, and is of substantial additive value because an anatomic coronary stenosis does not necessarily correspond to a reduction of coronary blood flow. MPRI can distinguish between normal subjects and patients with coronary artery disease (CAD) of either macrovascular or microvascular etiology (lesions in epicardial coronary arteries or micro-circulation defects, respectively) [13-15].

Previous stress CMR studies by our group and others have demonstrated perfusion abnormalities by means of MPRI in patients with cardiac syndrome X [16], Raynaud's phenomenon [17] and SLE [18], with a much higher sensitivity than conventional imaging modalities. The CE-MARC study showed also the superiority of CMR over single photon emission tomography (SPECT) for the detection of myocardial ischemia and fibrosis [19]. No comparison between APS patients and healthy controls has been carried out to date with regard to stress CMR findings [20].

Our aim was to examine myocardial perfusion defects using stress CMR in patients with PAPS and SLE/APS without known CAD, in comparison with age- and sex-matched healthy controls. We also evaluated potential associations between CMR findings and APS-related and classic CVD risk factors and coronary angiography findings.

\section{Methods}

\subsection{Study Participants}

Consecutive patients without any previous history of coronary artery disease (CAD) who met the updated Sapporo classification criteria for antiphospholipid syndrome (APS) [1] were recruited for participation in this study. Patients with SLE/APS fulfilled also the ACR classification criteria for systemic lupus erythematosus (SLE) [21]. Age- and sex-matched healthy controls were also recruited from a group of individuals participating in recreational sports. Exclusion criteria were prior atherosclerotic-origin cardiovascular disease (CVD), allergy to gadolinium, glomerular filtration rate $(\mathrm{GFR})<30 \mathrm{~mL} / \mathrm{min} / 1.75 \mathrm{~m}^{2}$, pregnancy or claustrophobia.

Demographic, clinical and laboratory characteristics of APS patients, and classic CVD risk factors, were recorded. Blood samples were collected at the time of examination and high-sensitivity CRP 
(hs-CRP) and high-sensitivity troponin T (hs-TnT) were measured by enzyme-linked immunosorbent assay (Elecsys 2010, Roche, Basel, Switzerland). All patients were followed up for 12 months, and clinically overt myocardial infarction (MI) or unstable angina was recorded based on physician adjudication. All participants provided informed consent and the protocol was approved by the Laikon Hospital Scientific Council.

\subsection{Cardiovascular Magnetic Resonance Imaging Technique}

All APS patients and matched healthy controls underwent a cardiovascular magnetic resonance (CMR) examination on a 1.5 Tesla scanner (Signa CV/i, GE Medical Systems) using ECG-triggered steady-state, free precession breath-hold cines (echo time (TE)/ repetition time (TR) 1.6/3.2 ms, flip angle 60) in long-axis planes and sequential $8 \mathrm{~mm}$ short-axis slices ( $3 \mathrm{~mm}$ gap) from the atrioventricular ring to the apex. Stress perfusion CMR was performed using a $140 \mathrm{mg} / \mathrm{kg} / \mathrm{min}$ adenosine infusion for $4 \mathrm{~min}[10,22]$ and a $0.05 \mathrm{mmol} / \mathrm{kg}$ Gd-DTPA bolus (Schering) was administered during the first-pass perfusion study (IR balanced Turbo Field Echo, TR $2.8 \mathrm{~ms}$, TE $1.38 \mathrm{~ms}$, FA 45, slice thickness $8 \mathrm{~mm}$, preparation pulse delay $200 \mathrm{~ms}$ ). A rest perfusion study was performed using the same protocol. Late gadolinium enhancement (LGE) images were acquired 10 min after the intravenous administration of an additional $0.1 \mathrm{mmol} / \mathrm{kg}$ of Gd-DTPA. Images were acquired in short-axis planes using an inversion-recovery gradient echo sequence for fibrosis detection (3D-Turbo field echo sequence, TR $5.1 \mathrm{~ms}$, TE $2.5 \mathrm{~ms}$, FA 15, slice thickness $8 \mathrm{~mm}$ ). Inversion times were adjusted to null normal myocardium (typically 320-440 ms; pixel size $1.7 \times 1.4 \mathrm{~mm}$ ).

\subsection{Cardiovascular Magnetic Resonance Analysis}

CMR scans were independently analyzed by two experienced observers blinded to the clinical data (SM, DH), and a consensus was used for discordant findings. The intra- and inter-observer variability was 0.88 and 0.85 , respectively. Ventricular volumes and function were measured for both ventricles using standard techniques and analyzed using specialized software [23,24]. Perfusion defects were assessed by both visual and parametric analysis. Quantification was performed using a delineation of endo and epicardial left ventricular (LV) borders throughout first-pass perfusion (MEDIS system, Leiden, The Netherlands). Stress and rest perfusion slopes were derived using Fermi-fitting of signal intensity vs time and normalized to the LV blood pool slope. Myocardial perfusion reserve index (MPRI) was calculated by dividing the median hyperemic MBF during stress by the median resting MBF [8]. Epicardial and endocardial contours of the LV myocardium for three short-axis slices (basal, mid and apical) were determined by the software, and manually corrected if needed to acquire intensity over time curves, which were used to measure the MPRI. The whole myocardial, subendocardial and subepicardial MPRI were calculated as the ratio of stress/rest relative perfusion up-slope, corrected for LV cavity up-slope. The subendocardial and subepicardial layers were automatically defined by the software as the inner and outer $50 \%$ of the wall thickness between the contoured myocardium. Finally, LGE images were assessed for midwall or subepicardial enhancement, compatible with microvascular disease $[25,26]$ and for subendocardial or transmural enhancement in the distribution of a coronary artery, suggestive of MI [27]. In the latter case, diagnostic coronary angiography was offered to the corresponding patient, without taking other clinical findings into account (e.g., exercise testing).

\subsection{Statistical Analysis}

Statistical analyses were performed with Stata v.15 SE. The normality of continuous variables was visually determined using Q-Q plots and/or histograms. Normally distributed variables are presented as mean (standard deviation), not-normally distributed continuous variables are presented as median (interquartile range), and binary variables are presented as $\mathrm{N}(\%)$.

Differences between APS patients and matched controls were investigated using paired-sample $t$-tests for continuous variables, sign tests for continuous not-normally distributed variables and McNemar's test for binary variables. For intra-group (non-paired) comparisons, normally distributed 
variables were compared with independent-sample $t$-tests, not-normally distributed variables with Mann-Whitney $U$ tests, and binary variables with chi-square tests. Linear regression analyses were used for multivariable corrections when investigating continuous outcomes. Statistical significance was considered for $p \leq 0.05$.

\section{Results}

Forty four patients with APS (22 with PAPS, 22 with SLE-APS) were included in the study (all Caucasian, $64 \%$ female, mean age: $44 \pm 13$ years, median disease duration: $12.0(5.5,21.0)$. One to one age- and sex-matching of 44 healthy controls without any history of cardiac disease was performed (mean age: $44 \pm 11$ years, 64\% female). Baseline characteristics of all APS patients, and comparisons between those with PAPS and SLE/APS, are presented in Table 1. Patients with PAPS had a significantly higher prevalence of a previous stroke of non-atherosclerotic origin [5 $(23 \%)$ vs. $0(0 \%) p=0.018]$ and recurrent thrombosis [12 (55\%) vs. $5(23 \%) p=0.030]$, compared to SLE/APS patients. Additionally, PAPS patients with detectable TnT (above the 99th percentile upper reference limit of $13.9 \mathrm{ng} / \mathrm{L}$ ) had significantly higher hs-TnT levels compared to those with SLE/APS and detectable TnT [19.5 (7.0-36.0) vs. $6.7(4.3-9.0), p=0.040]$, but the proportion of patients with hs-TnT below the detection limit did not differ significantly between the two groups [15 $(68 \%)$ vs. $12(55 \%), p=0.35]$. The median MPRI in the entire APS cohort was $1.48(0.9,1.9)$.

Table 1. Baseline demographic, clinical and cardiovascular magnetic resonance (CMR) characteristics of antiphospholipid syndrome (APS) patients.

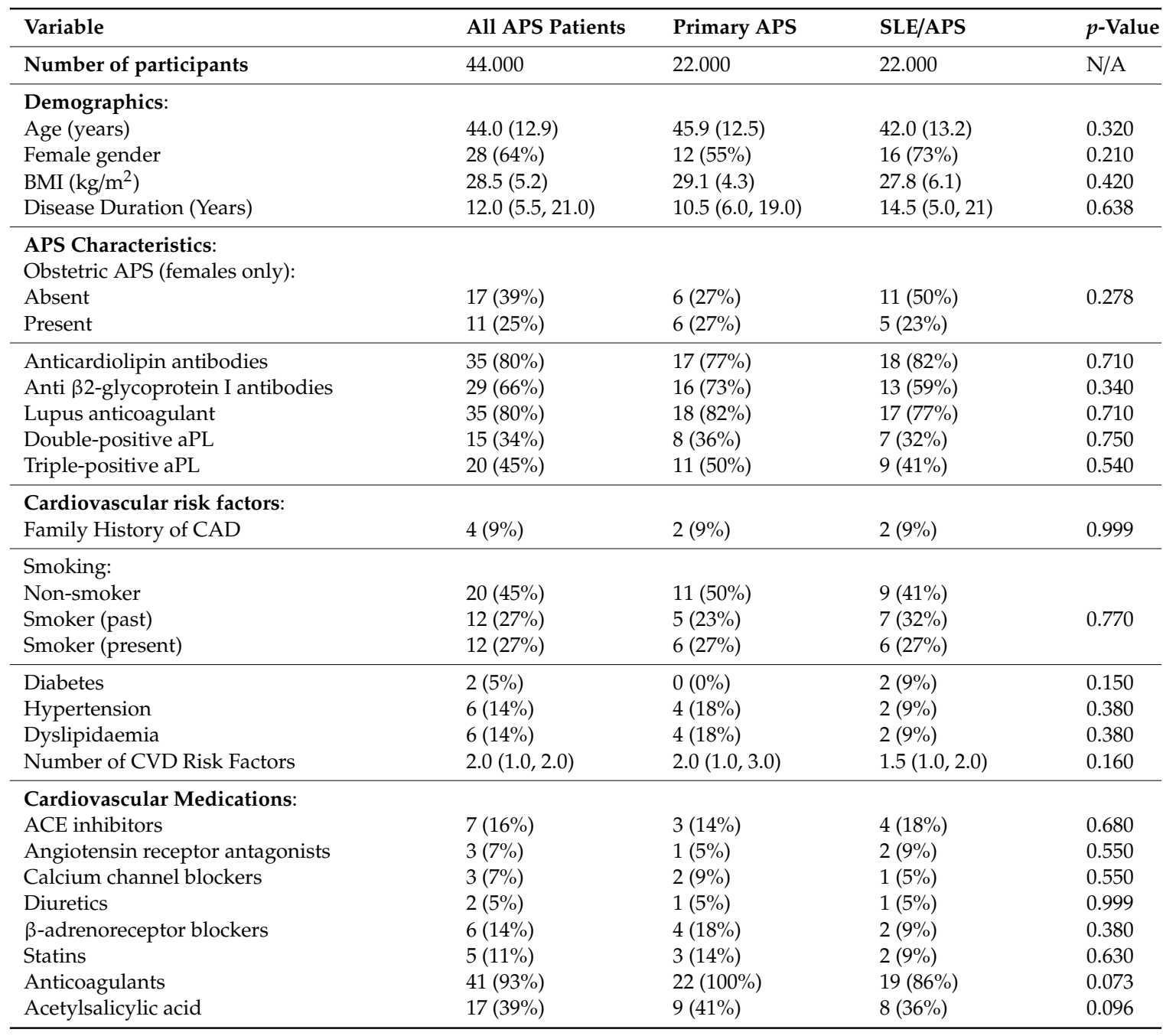


Table 1. Cont.

\begin{tabular}{|c|c|c|c|c|}
\hline Variable & All APS Patients & Primary APS & SLE/APS & $p$-Value \\
\hline Corticosteroids & $14(32 \%)$ & $1(5 \%)$ & $13(59 \%)$ & $<0.001$ \\
\hline Azathioprine & $5(11 \%)$ & $4(18 \%)$ & $1(5 \%)$ & 0.154 \\
\hline Methotrexate & $4(9 \%)$ & $1(5 \%)$ & $3(14 \%)$ & 0.290 \\
\hline Mycophenolate Mofetil & $4(9 \%)$ & $0(0 \%)$ & $4(18 \%)$ & 0.036 \\
\hline \multicolumn{5}{|l|}{ Previous Vascular Events: } \\
\hline Stroke & $5(11 \%)$ & $5(23 \%)$ & $0(0 \%)$ & 0.018 \\
\hline Arterial thrombosis & $19(43 \%)$ & $12(55 \%)$ & $7(32 \%)$ & 0.130 \\
\hline Venous thrombosis & $30(68 \%)$ & $15(68 \%)$ & $15(68 \%)$ & 0.999 \\
\hline Recurrent thrombosis & $17(39 \%)$ & $12(55 \%)$ & $5(23 \%)$ & 0.030 \\
\hline LV end systolic volume (mL) & $47.5(38.5,61.5)$ & $49.5(40.0,69.0)$ & $45.0(33.0,57.0)$ & 0.250 \\
\hline LV ejection fraction (\%) & $63.5(60.0,67.0)$ & $63.0(60.0,67.0)$ & $64.0(60.0,67.0)$ & 0.800 \\
\hline LV mass (g) & $81.0(65.5,98.5)$ & $86.0(67.0,106.0)$ & $78.0(60.0,89.0)$ & 0.150 \\
\hline RV end diastolic volume (mL) & $109.0(84.0,126.5)$ & $114.5(89.0,129.0)$ & $103.5(70.0,116.0)$ & 0.330 \\
\hline RV end systolic volume (mL) & $38.5(29.0,48.5)$ & $40.5(32.0,49.0)$ & $36.0(24.0,46.0)$ & 0.400 \\
\hline RV ejection fraction (\%) & $64.0(59.5,67.0)$ & $63.0(59.0,66.0)$ & $65.0(60.0,69.0)$ & 0.450 \\
\hline LGE (present/absent) & $16(36 \%)$ & $8(36 \%)$ & $8(36 \%)$ & 0.400 \\
\hline LGE as \% LV mass (only if LGE is present) & $4.5(3.5-7.5)$ & $6.5(3.0,15.0)$ & $4.0(3.5,5.5)$ & 0.290 \\
\hline Myocardial perfusion reserve index (MPRI) & $1.5(0.9,1.9)$ & $1.4(0.9,1.8)$ & $1.5(0.9,2.1)$ & 0.999 \\
\hline \multicolumn{5}{|l|}{ Biomarkers: } \\
\hline High-sensitivity C-reactive protein (mg/L) & $2.4(1.2,5.0)$ & $1.7(1.1,5.0)$ & $2.6(1.2,4.9)$ & 0.760 \\
\hline
\end{tabular}

Values represent the median and interquartile range mean \pm standard deviation (SD) for quantitative parameters and percent participants within each subgroup for qualitative characteristics. CMR = cardiovascular magnetic resonance; $\mathrm{BMI}=$ body mass index; APS = antiphospholipid syndrome; CAD = coronary artery disease; CVD = cardiovascular disease; LV = left ventricular; RV = right ventricular; LGE = late gadolinium enhancement, and here SLE = systemic lupus erythematosus.

Descriptive statistics and comparisons between the CMR indices of APS patients and their matched controls are presented in Table 2. The LV volumes and ejection fractions did not differ significantly between the two groups. However, APS patients had a significantly lower median LV mass compared to matched controls [81.0 (65.5-98.5) vs. 121.5 (112.0-140.0), $p<0.001$ ]. Median RVEDV and RVESV were also lower in APS patients compared to controls, but only RVEDV reached statistical significance [RVEDV: 109.0 (84.0-126.5) vs. 125.0 (120.0-150.0), $p<0.001$; RVESV 38.5 (29.0-48.5) vs. 45.0 (40.0-48.0), $p=0.057]$. There was a trend for higher median RVEF in APS patients compared to controls, but this also did not reach statistical significance $(p=0.079)$.

Median MPRI was significantly lower in APS patients compared to healthy controls [1.48 (0.9-1.9) vs. $2.7(2.2-3.2), p<0.001]$. Sixteen (36\%) APS patients had visible areas of myocardial scar, expressed as LGE, compared to none of the healthy controls $(p<0.001)$. Myocardial scar following the distribution of coronary arteries was identified in nine $(20 \%)$ patients (five in the anteroseptal and four in the inferolateral LV wall) (Figure 1A), while diffuse subendocardial fibrosis (DSF) (Figure 1B) was identified in seven $(16 \%)$ patients. In patients with a positive LGE, the median LGE values expressed as percentage of LV mass were 4.5 (3.5-7.5). Coronary angiography was performed in 12 of the 16 APS patients with positive LGE, with macrovascular (obstructive) CAD identified only in two patients, one of which subsequently underwent angioplasty of the left anterior descending artery. Three of 10 patients with otherwise normal coronary angiography findings had abnormal LVEF values $(<55 \%)$ and concomitant low MPRI values below the median of the APS group $(<1.48)$. 
Table 2. Comparison of CMR findings between APS patients and matched controls.

\begin{tabular}{cccc}
\hline Parameters & $\begin{array}{c}\text { Descriptive Statistics } \\
\text { (APS Patients) }\end{array}$ & $\begin{array}{c}\text { Descriptive Statistics } \\
\text { (Matched Controls) }\end{array}$ & $p$-Value \\
\hline Number of participants & 44 & 44 & N/A \\
\hline LVEDV $(\mathrm{mL})$ & $131.5(110.0,160.5)$ & $140.0(123.0,160.0)$ & 0.628 \\
\hline LVESV $(\mathrm{mL})$ & $47.5(38.5,61.5)$ & $51.0(44.0,63.5)$ & 0.517 \\
\hline LVEF $(\%)$ & $63.5(60.0,67.0)$ & $63.0(58.0,66.0)$ & 0.607 \\
\hline LV Mass $(\mathrm{g})$ & $81.0(65.5,98.5)$ & $121.5(112.0,140.0)$ & $<0.001$ \\
\hline RVEDV $(\mathrm{mL})$ & $109.0(84.0,126.5)$ & $125.0(120.0,150.0)$ & $<0.001$ \\
\hline RVESV $(\mathrm{mL})$ & $38.5(29.0,48.5)$ & $45.0(40.0,48.0)$ & 0.057 \\
\hline RVEF $(\%)$ & $64.0(59.5,67.0)$ & $60.0(58.0,64.0)$ & 0.079 \\
\hline LGE & $16(36 \%)$ & $0(0 \%)$ & $<0.001$ \\
\hline MPRI & $1.5(0.9,1.9)$ & $2.7(2.2,3.2)$ & $<0.001$ \\
\hline
\end{tabular}

These values represent the median and interquartile range. CMR = Cardiovascular Magnetic Resonance; APS = antiphospholipid syndrome; LVEDV = left ventricular end diastolic volume; LVESV = left ventricular end systolic volume; $L V E F=$ left ventricular ejection fraction; $L V=$ mass left ventricular mass; $R V E D V=$ right ventricular end diastolic volume; RVESV = right ventricular end systolic volume; RVEF = right ventricular ejection fraction; $\mathrm{LGE}=$ late gadolinium enhancement; MPRI = myocardial perfusion reserve index .

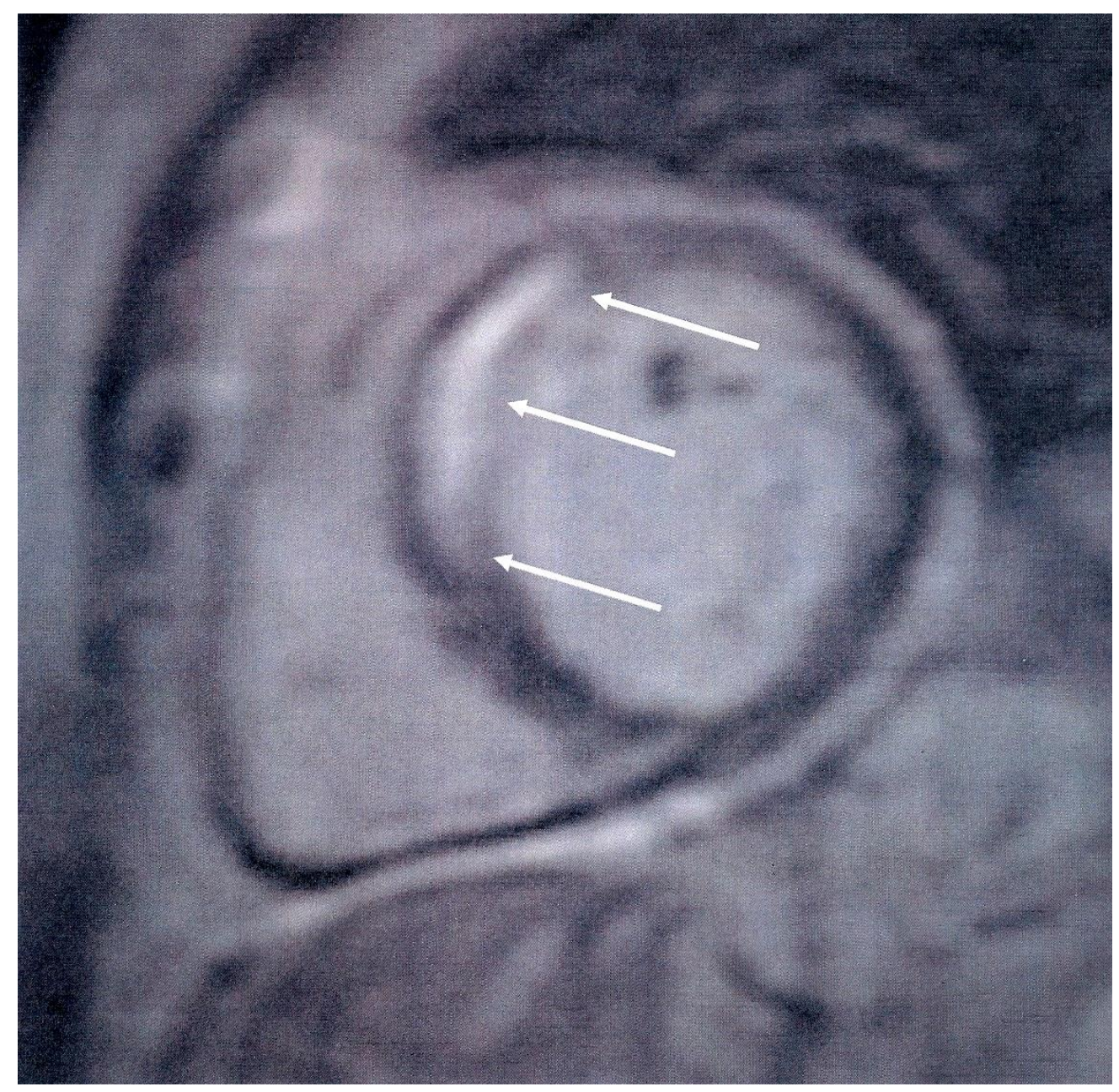

(A)

Figure 1. Cont. 


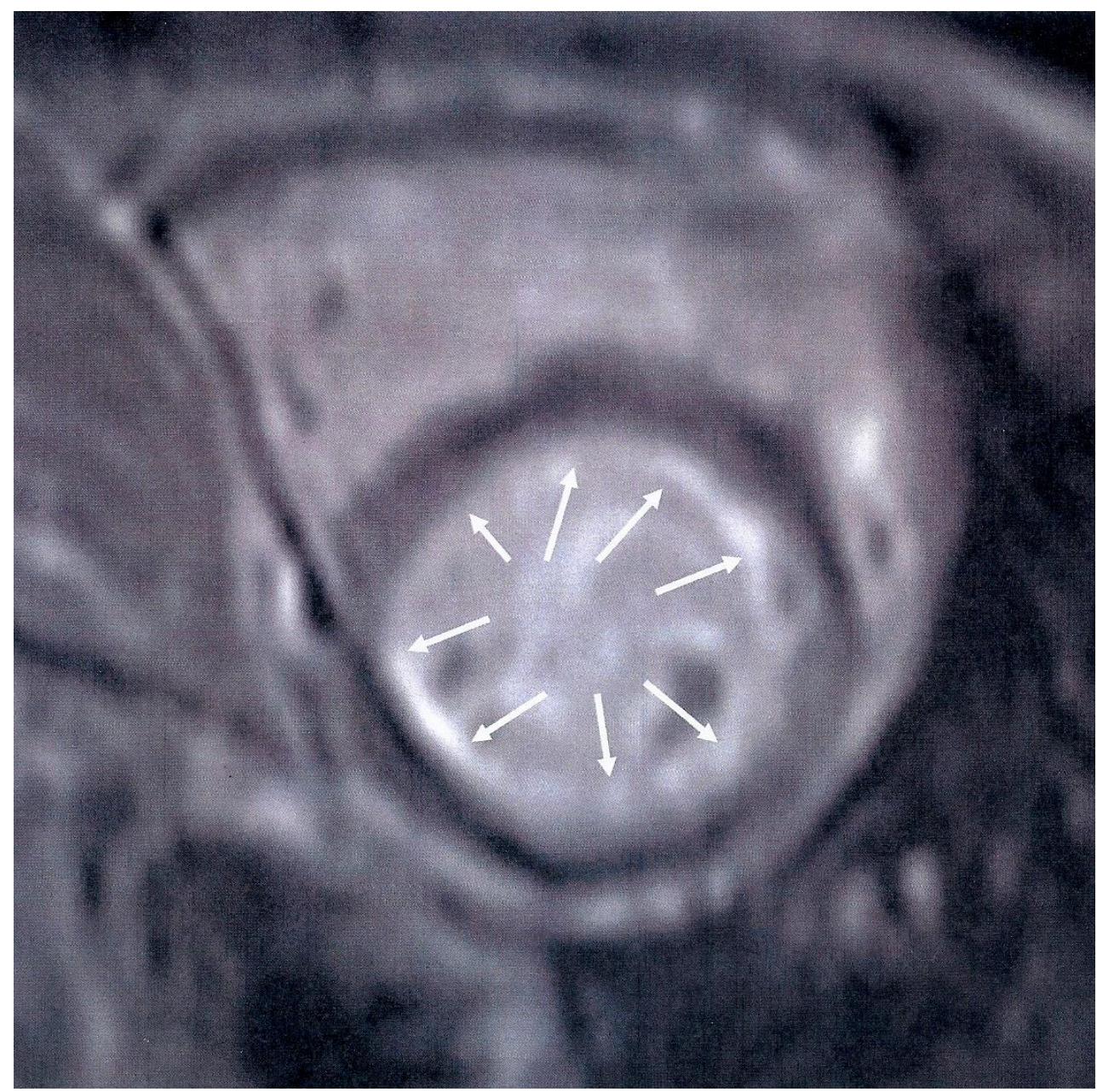

(B)

Figure 1. (A) Short-axis inversion recovery sequence showing septal myocardial scar (arrows), following the distribution of the left anterior descending artery, indicative of myocardial infarction. (B) Short-axis inversion recovery sequence showing diffuse subendocardial scar (arrows) due to microvascular disease.

When comparing patients with MPRI above and below the median value of the APS group (Table 3), no significant differences were identified in any demographic, clinical, laboratory and CMR characteristics, with the exception of obstetric APS and LVEF. The former was significantly less prevalent in patients with higher MPRI values [3 (15\%) vs. $8(33 \%), p=0.029$ ], and the latter was significantly higher in patients with higher MPRI values $[61.5(58.5,65.0)$ vs. $64.5(62.5,68.0), p=0.041]$, albeit still largely within normal limits ( $>55 \%)$. Additionally, more male patients had a lower MPRI, but this did not reach statistical significance [11 $(46 \%)$ vs. $5(75 \%), p=0.153]$. Finally, there was a trend for higher MPRI values in patients receiving treatment with corticosteroids compared to those not treated with corticosteroids, but this did not reach any statistical significance [9 (45\%) vs. $5(21 \%)$, $p=0.087]$. A similar non-significant trend for higher MPRI was identified in patients treated with acetylsalicylic acid (ASA, Aspirin $100 \mathrm{mg}$ daily) [12 (50\%) vs. $5(25 \%), p=0.09$ ]. No association with hydroxychloroquine use, or hsCRP and hsTnT levels was detected. Of the aforementioned variables, those with a $p$-value $<0.1$ were included in a multivariable linear regression model for predicting MPRI values. Obstetric APS was replaced with female gender, as it would preclude the analysis of male patients. However, this did not lead to any statistically significant results (Table 4). No significant associations were also found between LGE and APS characteristics, classic CVD risk factors, or the hsCRP and hsTnT levels, in both descriptive (Table 5) and multivariate analysis. 
Table 3. Comparison of patients with MPRI above and below the median of the group (=1.48).

\begin{tabular}{|c|c|c|c|}
\hline Variable & MPRI below Median & MPRI above Median & $p$-Value \\
\hline Number of participants & 24 & 20 & \\
\hline \multicolumn{4}{|l|}{ Demographics: } \\
\hline Age (years) & $43.4(13.3)$ & $44.7(12.6)$ & 0.750 \\
\hline Female gender & $13(54 \%)$ & $15(75 \%)$ & 0.153 \\
\hline BMI $\left(\mathrm{kg} / \mathrm{m}^{2}\right)$ & $27.6(5.9)$ & $29.5(4.2)$ & 0.230 \\
\hline Disease Duration (Years) & $14.0(6.5,21.0)$ & $9.0(4.0,20.0)$ & 0.395 \\
\hline \multicolumn{4}{|l|}{ APS Characteristics: } \\
\hline Primary APS & $13(54 \%)$ & $9(45 \%)$ & 0.540 \\
\hline \multicolumn{4}{|l|}{ Obstetric APS (females only): } \\
\hline Absent & $5(21 \%)$ & $12(60 \%)$ & 0.029 \\
\hline Present & $8(33 \%)$ & $3(15 \%)$ & \\
\hline Anticardiolipin antibodies & $18(75 \%)$ & $17(85 \%)$ & 0.410 \\
\hline anti $\beta 2$-glycoprotein I antibodies & $15(63 \%)$ & $14(70 \%)$ & 0.600 \\
\hline Lupus anticoagulant & $20(83 \%)$ & $15(75 \%)$ & 0.500 \\
\hline Double-positive aPL & $7(29 \%)$ & $8(40 \%)$ & 0.450 \\
\hline Triple-positive aPL & $11(46 \%)$ & $9(45 \%)$ & 0.960 \\
\hline \multicolumn{4}{|l|}{ Cardiovascular risk factors: } \\
\hline Family History of CAD & $2(8 \%)$ & $2(10 \%)$ & 0.850 \\
\hline \multicolumn{4}{|l|}{ Smoking: } \\
\hline Non-smoker & $10(42 \%)$ & $10(50 \%)$ & \\
\hline Smoker (past) & $6(25 \%)$ & $6(30 \%)$ & 0.610 \\
\hline Smoker (present) & $8(33 \%)$ & $4(20 \%)$ & \\
\hline Diabetes & $1(4 \%)$ & $1(5 \%)$ & 0.890 \\
\hline Hypertension & $3(13 \%)$ & $3(15 \%)$ & 0.810 \\
\hline Dyslipidaemia & $2(8 \%)$ & $4(20 \%)$ & 0.260 \\
\hline Number of CVD Risk Factors & $2.0(0.0,2.0)$ & $2.0(1.0,2.0)$ & 0.300 \\
\hline \multicolumn{4}{|l|}{ Cardiovascular Medications: } \\
\hline ACE inhibitors & $4(17 \%)$ & $3(15 \%)$ & 0.880 \\
\hline Angiotensin receptor antagonists & $1(4 \%)$ & $2(10 \%)$ & 0.440 \\
\hline Calcium channel blockers & $2(8 \%)$ & $1(5 \%)$ & 0.660 \\
\hline Diuretics & $2(8 \%)$ & $0(0 \%)$ & 0.190 \\
\hline$\beta$-Adrenoreceptor blockers & $4(17 \%)$ & $2(10 \%)$ & 0.520 \\
\hline Statins & $2(8 \%)$ & $3(15 \%)$ & 0.490 \\
\hline Anticoagulants & $23(96 \%)$ & $18(90 \%)$ & 0.440 \\
\hline Acetylsalicylic acid & $12(50 \%)$ & $5(25 \%)$ & 0.090 \\
\hline \multicolumn{4}{|l|}{ Immunosupressive Medications: } \\
\hline Corticosteroids & $5(21 \%)$ & $9(45 \%)$ & 0.087 \\
\hline Hydroxychloroquine & $13(54 \%)$ & $13(65 \%)$ & 0.470 \\
\hline Azathioprine & $2(8 \%)$ & $2(8 \%)$ & 0.488 \\
\hline Methotrexate & $3(13 \%)$ & $1(5 \%)$ & 0.390 \\
\hline Mycophenolate Mofetil & $2(8 \%)$ & $2(10 \%)$ & 0.850 \\
\hline Mycophenolic Acid & $0(0 \%)$ & $1(5 \%)$ & 0.270 \\
\hline \multicolumn{4}{|l|}{ Previous Vascular Events: } \\
\hline Stroke & $2(8 \%)$ & $3(14 \%)$ & 0.490 \\
\hline Arterial thrombosis & $10(42 \%)$ & $9(45 \%)$ & 0.820 \\
\hline Venous thrombosis & $15(63 \%)$ & $15(75 \%)$ & 0.380 \\
\hline Recurrent thrombosis & $9(38 \%)$ & $8(40 \%)$ & 0.870 \\
\hline Recurrence on Anticoagulants & $5(21 \%)$ & $6(32 \%)$ & 0.420 \\
\hline \multicolumn{4}{|l|}{ CMR Parameters: } \\
\hline Left ventricular end diastolic volume (mL) & $129.0(112.5,158.5)$ & $142.5(110.0,162.0)$ & 0.800 \\
\hline Left ventricular end systolic volume (mL) & $48.0(39.5,66.5)$ & $47.0(38.5,60.5)$ & 0.690 \\
\hline Left ventricular ejection fraction $(\%)$ & $61.5(58.5,65.0)$ & $64.5(62.5,68.0)$ & 0.041 \\
\hline Left ventricular mass (g) & $88.0(66.5,103.0)$ & $76.0(59.5,84.0)$ & 0.110 \\
\hline Right ventricular end diastolic volume (mL) & $103.5(79.0,124.0)$ & $115.5(84.5,129.5)$ & 0.500 \\
\hline Right ventricular end systolic volume $(\mathrm{mL})$ & $38.5(29.5,48.5)$ & $38.5(28.5,48.5)$ & 0.970 \\
\hline Right ventricular ejection fraction (\%) & $62.5(56.0,66.0)$ & $65.0(62.0,69.0)$ & 0.073 \\
\hline LGE as \% LV mass (only if LGE is present) & $5.0(3.0,8.0)$ & $4.0(4.0,7.0)$ & 0.950 \\
\hline Late gadolinium enhancement (present/absent) & $11(46 \%)$ & $5(25 \%)$ & 0.150 \\
\hline \multicolumn{4}{|l|}{ Biomarkers: } \\
\hline High-sensitivity C-reactive protein (mg/L) & $3.0(0.8,5.8)$ & $2.2(1.2,2.7)$ & 0.350 \\
\hline High-sensitivity Troponin-T below lowest limit of detection & $14(58 \%)$ & $13(65 \%)$ & 0.650 \\
\hline High-sensitivity Troponin-T (pg/mL)-within detection range & $7.3(4.3,21.3)$ & $9.0(5.8,19.5)$ & 0.490 \\
\hline
\end{tabular}

Values represent the median and interquartile range mean \pm standard deviation (SD) for quantitative parameters and percent participants within each subgroup for qualitative characteristics. MPRI = myocardial perfusion reserve index; $\mathrm{BMI}=$ body mass index; $\mathrm{APS}=$ antiphospholipid syndrome; $\mathrm{CAD}=$ coronary artery disease; $\mathrm{CVD}=$ cardiovascular disease 
Table 4. Multivariable linear regression analysis for predicting the MPRI values. Examined covariates were selecting on the basis of achieving a $p$-value $<0.1$ between low and high MPRI.

\begin{tabular}{lll}
\hline Variable & Coefficient $[95 \%$ Confidence Interval] & $p$-Value \\
\hline Female gender & $-0.0167[-0.48,0.45]$ & 0.943 \\
LVEF $(\%)$ & $-0.0081[-0.024,0.041]$ & 0.615 \\
RVEF $(\%)$ & $0.024[-0.18,0.067]$ & 0.251 \\
Corticosteroids & $0.445[-0.027,0.918]$ & 0.064 \\
Acetylsalicylic acid & $-2.651[-0.67,0.14]$ & 0.196 \\
\hline
\end{tabular}

MPRI = myocardial perfusion reserve index; $\mathrm{LVEF}$ = left ventricular ejection fraction; RVEF = right ventricular ejection fraction.

Table 5. Comparison of patients with and without late gadolinium enhancement (LGE).

\begin{tabular}{|c|c|c|c|}
\hline Variable & LGE Absent & LGE Present & $p$-Value \\
\hline Number of participants & 28 & 16 & \\
\hline \multicolumn{4}{|l|}{ Demographics: } \\
\hline Age (years) & $44.4(12.8)$ & $43.1(13.3)$ & 0.750 \\
\hline Female gender & $18(64 \%)$ & $10(63 \%)$ & 0.906 \\
\hline BMI $\left(\mathrm{kg} / \mathrm{m}^{2}\right)$ & $29.3(4.9)$ & $27.0(5.6)$ & 0.170 \\
\hline Disease Duration (Years) & $10.5(6.5,20.5)$ & $14.0(4.5,22.0)$ & 0.660 \\
\hline \multicolumn{4}{|l|}{ APS Characteristics: } \\
\hline Primary APS & $14(50 \%)$ & $8(50 \%)$ & 0.999 \\
\hline \multicolumn{4}{|l|}{ Obstetric APS (females only): } \\
\hline Absent & $9(32 \%)$ & $8(50 \%)$ & 0.298 \\
\hline Present & $9(32 \%)$ & $2(12.5 \%)$ & \\
\hline Anticardiolipin antibodies & $23(82 \%)$ & $12(75 \%)$ & 0.570 \\
\hline Anti $\beta 2$-glycoprotein I antibodies & $21(75 \%)$ & $8(50 \%)$ & 0.092 \\
\hline Lupus anticoagulant & $21(75 \%)$ & $14(88 \%)$ & 0.320 \\
\hline Double-positive aPL & $11(39 \%)$ & $4(25 \%)$ & 0.336 \\
\hline Triple-positive aPL & $13(46 \%)$ & $7(44 \%)$ & 0.860 \\
\hline \multicolumn{4}{|l|}{ Cardiovascular risk factors: } \\
\hline Family History of CAD & $2(7 \%)$ & $2(13 \%)$ & 0.550 \\
\hline \multicolumn{4}{|l|}{ Smoking: } \\
\hline Non-smoker & $10(36 \%)$ & $10(63 \%)$ & \\
\hline Smoker (past) & $11(39 \%)$ & $1(6 \%)$ & 0.054 \\
\hline Smoker (present) & $7(25 \%)$ & $5(31 \%)$ & \\
\hline Diabetes & $1(4 \%)$ & $1(6 \%)$ & 0.680 \\
\hline Hypertension & $5(18 \%)$ & $1(6 \%)$ & 0.280 \\
\hline Dyslipidaemia & $4(14 \%)$ & $2(13 \%)$ & 0.870 \\
\hline Number of CVD Risk Factors & $2.0(1.0,2.0)$ & $1.0(0.0,2.0)$ & 0.029 \\
\hline \multicolumn{4}{|l|}{ Cardiovascular Medications: } \\
\hline ACE inhibitors & $3(11 \%)$ & $4(25 \%)$ & 0.210 \\
\hline Angiotensin receptor antagonists & $3(11 \%)$ & $0(0 \%)$ & 0.170 \\
\hline Calcium channel blockers & $2(7 \%)$ & $1(6 \%)$ & 0.910 \\
\hline Diuretics & $0(0 \%)$ & $2(13 \%)$ & 0.056 \\
\hline$\beta$-Adrenoreceptor blockers & $2(7 \%)$ & $4(25 \%)$ & 0.097 \\
\hline Statins & $4(14 \%)$ & $1(6 \%)$ & 0.420 \\
\hline Anticoagulants & $26(93 \%)$ & $15(94 \%)$ & 0.910 \\
\hline Acetylsalicylic acid & $12(43 \%)$ & $5(31 \%)$ & 0.450 \\
\hline \multicolumn{4}{|l|}{ Immunosupressive Medications: } \\
\hline Corticosteroids & $9(32 \%)$ & $5(31 \%)$ & 0.950 \\
\hline Hydroxychloroquine & $16(57 \%)$ & $10(63 \%)$ & 0.730 \\
\hline Azathioprine & $2(\%)$ & $1(\%)$ & 0.910 \\
\hline Methotrexate & $3(11 \%)$ & $1(6 \%)$ & 0.620 \\
\hline Mycophenolate Mofetil & $0(0 \%)$ & $1(6 \%)$ & 0.180 \\
\hline Mycophenolic Acid & $2(7 \%)$ & $2(13 \%)$ & 0.550 \\
\hline
\end{tabular}


Table 5. Cont.

\begin{tabular}{|c|c|c|c|}
\hline Variable & LGE Absent & LGE Present & $p$-Value \\
\hline \multicolumn{4}{|l|}{ Previous Vascular Events: } \\
\hline Stroke & $4(\%)$ & $1(\%)$ & 0.420 \\
\hline Arterial thrombosis & $14(50 \%)$ & $5(31 \%)$ & 0.230 \\
\hline Venous thrombosis & $18(64 \%)$ & $12(75 \%)$ & 0.460 \\
\hline Recurrent thrombosis & $12(43 \%)$ & $5(31 \%)$ & 0.450 \\
\hline Recurrence on Anticoagulants & $7(26 \%)$ & $4(25 \%)$ & 0.950 \\
\hline \multicolumn{4}{|l|}{ CMR Parameters: } \\
\hline Left ventricular end diastolic volume $(\mathrm{mL})$ & $126.5(104.5,147.5)$ & $158.5(123.5,185.5)$ & 0.071 \\
\hline Left ventricular end systolic volume (mL) & $47.5(36.5,55.0)$ & $52.0(41.5,80.5)$ & 0.120 \\
\hline Left ventricular ejection fraction $(\%)$ & $64.0(61.0,66.5)$ & $61.0(53.0,67.5)$ & 0.240 \\
\hline Left ventricular mass $(\mathrm{g})$ & $77.0(61.5,95.0)$ & $85.5(67.0,107.0)$ & 0.280 \\
\hline Right ventricular end diastolic volume (mL) & $110.0(84.0,126.5)$ & $104.5(80.0,127.0)$ & 0.970 \\
\hline Right ventricular end systolic volume (mL) & $38.5(28.5,49.5)$ & $38.5(30.0,46.5)$ & 0.760 \\
\hline Right ventricular ejection fraction (\%) & $65.0(60.5,68.5)$ & $62.5(55.0,65.5)$ & 0.092 \\
\hline \multicolumn{4}{|l|}{ Biomarkers: } \\
\hline High-sensitivity C-reactive protein (mg/L) & $2.2(1.1,3.3)$ & $3.5(1.2,5.6)$ & 0.407 \\
\hline High-sensitivity Troponin-T below lowest limit of detection & $18(64 \%)$ & $9(56 \%)$ & 0.600 \\
\hline High-sensitivity Troponin- $\mathrm{T}(\mathrm{pg} / \mathrm{mL})$-within detection range & $7.3(5.6,18.4)$ & $9.0(4.6,36.0)$ & 0.380 \\
\hline
\end{tabular}

At the twelve month follow-up, three patients (6.8\%) experienced CAD complications (1 MI, and 2 unstable angina), importantly those with the lowest MPRI values $(0.48,0.56$ and 0.58 , respectively). However, meaningful statistical analyses could not be performed due to small numbers.

\section{Discussion}

This is the first study to our knowledge that evaluated the presence of silent myocardial ischemia and fibrosis by stress CMR in patients with APS. The most striking finding of our study is the detection of highly-reduced MPRI compared with age- and sex-matched healthy controls, irrespective of the presence of LGE. Myocardial fibrosis, expressed as LGE, was detected in one third of APS patients, of which only two patients had abnormal coronary angiography findings. These findings demonstrate that even in asymptomatic APS patients without evidence of fibrosis or macrovascular CAD detected by coronary angiography, silent myocardial ischemia may exist.

We also identified for the first time a significantly reduced LV mass in patients with APS compared to controls. Although it may be argued that the athletic activities followed by the healthy controls in our study might lead to LV mass increase, the actual difference between the groups is larger than what might reasonably be explained by exercise-induced LV hypertrophy alone [28]. On the other hand, little is known about myocardial mass changes preceding clinically overt cardiac involvement. According to currently published literature, reduced LV mass has been demonstrated in patients with rheumatoid arthritis, and was considered a predisposing factor for developing future heart failure [29]. Nevertheless, evidence is lacking about which factors can influence LV mass in APS apart from hemodynamics.

Myocardial perfusion defects in APS patients without prior CAD were previously examined by three studies using contrast echocardiography and scintigraphy with radionuclides [30], SPECT [31] and N-ammonia PET [32], respectively. Perfusion abnormalities were detected in 30\%, 57.7\% and 38.8\% of 11,26 , and 18 patients tested, respectively. The limitations of these studies were the small number of included patients, the lack of comparison groups, and the use of imaging modalities of inferior diagnostic performance for myocardial perfusion assessment compared to CMR [14,22]. The only study that used CMR in APS showed a significantly higher prevalence of silent myocardial fibrosis compared to healthy controls [12]. No previous study examined silent myocardial ischemia using stress CMR imaging. 
Our findings have important clinical implications for stratifying patients with APS at risk for heart disease. The early detection of reduced MPRI can be either due to macro- or micro-vascular CAD. If there is evidence of macro-vascular CAD, presenting as reduced MPRI and/or positive LGE following the distribution of coronary arteries, coronary angiography is indicated with subsequent standard interventional treatment, as needed. Microvascular disease on the other hand, presents as equally reduced MPRI in all myocardial territories with/or without DSF. In our study, we identified only two patients with macrovascular CAD, with the majority having microvascular CAD instead. Next to the aforementioned implications of low MPRI for microvascular CAD, the assessment of myocardial scar in asymptomatic APS patients has also important clinical implications for macrovascular CAD.

Firstly, it carries a prognostic value for the development of future arrhythmia [33] and/or heart failure [34] and secondly, it motivates and can justify further investigation with coronary angiography [34]. Finally, it may prompt initiation of specific medication use including ACE-inhibitors, $\beta$-adrenoreceptor blockers or aspirin [35].

These findings, in addition to the development of CAD events over a 12-month follow-up in three APS patients with the lowest MPRI at CMR, have also important therapeutic implications in APS. Given that almost all patients with APS were on adequate treatment with vitamin K antagonists, this finding raises questions about the presumed efficacy of currently employed anticoagulation strategies (as monotherapy) in criteria and non-criteria APS manifestations [36,37], and the potential need for additional cardioprotective treatment in patients with reduced MPRI [20]. In diabetic patients with abnormal MPRI without obstructive CAD, the use of antianginal agents was shown to improve microvascular ischemia [38], however, these effects in APS should be independently investigated in well-designed prospective studies. Lifestyle modification and aggressive monitoring and management of classic CVD risk factors are included in the general measures for the management of APS in adults $[39,40]$.

Interestingly, no significant associations were detected in our study between abnormal MPRI or LGE and APS clinical and laboratory characteristics or traditional CVD risk factors. A previous study [12] showed a trend between myocardial scarring and APS features, such as disease duration and positivity for anti- $\beta 2$-glycoprotein I antibodies, however, these associations did not reach statistical significance. In addition, we found no association between reduced MPRI and hs-TnT or hs-CRP levels. In a recent study of SLE patients [41], hs-TnT was significantly associated with myocardial edema detected by T2 mapping that was not evaluated by our stress CMR protocol. Patients receiving Aspirin treatment and those on corticosteroids tended to have higher MPRI values, but these trends did not reach statistical significance. However, inferences about these associations cannot be accurately drawn due to the relatively small number of our study population.

In addition to adenosine stress CMR use for the detection of silent perfusion abnormalities in APS, other strengths of the study are the comparison of CMR indices with coronary angiography findings in patients with abnormal LGE, and the 12-month follow-up of patients for CAD events. Our study has also some limitations. The relatively small number of patients might have precluded the identification of significant associations between MPRI and APS-related or classic CVD risk factors. Furthermore, novel CMR indices for the detection of diffuse myocardial edema and fibrosis (T1, T2 mapping and extracellular volume fraction quantification) [20] were not available in our department at the time of the CMR examination. In addition, coronary angiography was performed only in patients with an LGE presence. It could thus be argued that an unidentified macrovascular obstructive component in the coronary circulation might also be at work, given that microvascular disease is not the sole process leading to MPRI reductions without concomitant fibrosis.

In conclusion, silent myocardial ischemia and myocardial fibrosis are common in APS, independent of APS-related and classic CVD risk factors, hs-CRP and hs-TnT levels or coronary angiography findings. Our results support the diagnostic value of the CMR examination for the detection of silent microor macro-vascular CAD in APS. Early identification of ischemic heart lesions by CMR may motivate further cardiac investigation and an early initiation of cardioprotective treatment. In addition, our 
findings raise questions about the efficacy of currently-used therapeutic approaches for the prevention of CAD in APS, underlining the need for a re-evaluation of current practices by future studies.

Author Contributions: M.G.T. had full access to all the data in the study and takes responsibility for the integrity of the data and the accuracy of the data analysis. Study concept and design: M.G.T., S.I.M. Acquisition, analysis, or interpretation of data: M.G.T., S.I.M., P.P.S., G.M. Drafting of the manuscript: M.G.T., S.I.M. Critical revision of the manuscript and approval of the manuscript: S.I.M., M.T., O.K., K.T., P.A., S.V., G.K., D.A., D.H., P.S., M.G.T.

Funding: This work was supported by a Research Grant from the Greek Rheumatology Society and Professional Association of Rheumatologists, and the Special Account for Research Grants of the National and Kapodistrian University of Athens Medical School, Greece (Grant no. 11123).

Conflicts of Interest: Authors declare no conflict of interest.

\section{Abbreviations}

$\begin{array}{ll}\text { APS } & \text { antiphospholipid syndrome } \\ \text { aPL } & \text { antiphospholipid antibodies } \\ \text { CMR } & \text { cardiovascular magnetic resonance } \\ \text { CAD } & \text { coronary artery disease } \\ \text { CVD } & \text { cardiovascular disease } \\ \text { MPRI } & \text { Myocardial perfusion reserve index } \\ \text { LGE } & \text { late gadolinium enhancement } \\ \text { MBF } & \text { myocardial blood flow } \\ \text { LV } & \text { left ventricular } \\ \text { LVEDV } & \text { left ventricular end diastolic volume } \\ \text { LVESV } & \text { left ventricular end systolic volume } \\ \text { LVEF } & \text { left ventricular ejection fraction } \\ \text { RVEDV } & \text { right ventricular end diastolic volume } \\ \text { RVESV } & \text { right ventricular end systolic volume } \\ \text { RVEF } & \text { right ventricular ejection fraction } \\ \text { hs-CRP } & \text { high-sensitivity CRP } \\ \text { hs-TnT } & \text { high-sensitivity troponin T }\end{array}$

\section{References}

1. Miyakis, S.; Lockshin, M.D.; Atsumi, T.; Branch, D.W.; Brey, R.L.; Cervera, R.H.W.M.; Derksen, R.H.; de Groot, P.G.; Koike, T.; Meroni, P.L.; et al. International consensus statement on an update of the classification criteria for definite antiphospholipid syndrome (APS). J. Thromb. Haemost. 2006, 4, 295-306. [CrossRef] [PubMed]

2. Cervera, R.; Serrano, R.; Pons-Estel, G.J.; Ceberio-Hualde, L.; Shoenfeld, Y.; de Ramón, E.; Buonaiuto, V.; Jacobsen, S.; Zeher, M.M.; Tarr, T.; et al. Morbidity and mortality in the antiphospholipid syndrome during a 10-year period: A multicentre prospective study of 1000 patients. Ann. Rheum. Dis. 2015, 74, 1011-1018. [CrossRef] [PubMed]

3. Denas, G.; Jose, S.P.; Bracco, A.; Zoppellaro, G.; Pengo, V. Antiphospholipid syndrome and the heart: A case series and literature review. Autoimmun. Rev. 2015, 14, 214-222. [CrossRef] [PubMed]

4. Cervera, R.; Tektonidou, M.G.; Espinosa, G.; Cabral, A.R.; González, E.B.; Erkan, D.; Vadya, S.; Adrogué, H.E.; Solomon, M.; Zandman-Goddard, G.; et al. Task Force on Catastrophic Antiphospholipid Syndrome (APS) and Non-criteria APS Manifestations (I): Catastrophic APS, APS nephropathy and heart valve lesions. Lupus 2011, 20, 165-173. [CrossRef] [PubMed]

5. Kampolis, C.; Tektonidou, M.; Moyssakis, I.; Tzelepis, G.E.; Moutsopoulos, H.; Vlachoyiannopoulos, P.G. Evolution of cardiac dysfunction in patients with antiphospholipid antibodies and/or antiphospholipid syndrome: A 10-year followup study. Semin. Arthritis Rheum. 2014, 43, 558-565. [CrossRef]

6. Yedlapati, N.; Paladugu, N.; Spevack, D.M. Prinzmetal's angina in patients with antiphospholipid syndrome. Coron. Artery Dis. 2011, 22, 537-539. [CrossRef] [PubMed]

7. Sangle, S.R.; D'Cruz, D.P.; Syndrome, X. (angina pectoris with normal coronary arteries) and myocardial infarction in patients with anti-phospholipid (Hughes) syndrome. Lupus 2008, 17, 83-85. [CrossRef] 
8. Onea, R.; Germain, P.; Zimmermann, A. Coronary microvasculopathy and intracardiac thrombosis in antiphospholipid syndrome. Arch. Cardiovasc. Dis. 2012, 105, 461-462. [CrossRef]

9. Azeem, T.; Vassallo, M.; Samani, N.J. Images in cardiology. Endomyocardial fibrosis associated with antiphospholipid syndrome. Heart 2000, 84, 156. [CrossRef]

10. American College of Cardiology Foundation Task Force on Expert Consensus Documents; Hundley, W.G.; Bluemke, D.A.; Finn, J.P.; Flamm, S.D.; Fogel, M.A.; Friedrich, M.G.; Ho, V.B.; Jerosch-Herold, M.; Kramer, C.M.; et al. CCF/ACR/AHA/NASCI/SCMR 2010 expert consensus document on cardiovascular magnetic resonance: A report of the American College of Cardiology Foundation Task Force on Expert Consensus Documents. J. Am. Coll. Cardiol. 2010, 121, 2462-2508.

11. Mavrogeni, S.I.; Sfikakis, P.P.; Koutsogeorgopoulou, L.; Markousis-Mavrogenis, G.; Dimitroulas, T.; Kolovou, G.; Kitas, G.D. Cardiac Tissue Characterization and Imaging in Autoimmune Rheumatic Diseases. JACC Cardiovasc. Imaging 2017, 10, 1387-1396. [CrossRef] [PubMed]

12. Sacré, K.; Brihaye, B.; Hyafil, F.; Serfaty, J.M.; Escoubet, B.; Zennaro, M.C.; Lidove, O.; Laissy, J.P.; Papo, T. Asymptomatic myocardial ischemic disease in antiphospholipid syndrome: A controlled cardiac magnetic resonance imaging study. Arthritis Rheum. 2010, 62, 2093-2100. [PubMed]

13. Ponte, M.; Bettencourt, N.; Pereira, E.; Ferreira, N.D.; Chiribiri, A.; Schuster, A.; Albuquerque, A.; Gama, V.; Nagel, E. Anatomical versus functional assessment of coronary artery disease: Direct comparison of computed tomography coronary angiography and magnetic resonance myocardial perfusion imaging in patients with intermediate pre-test probability. Int. J. Cardiovasc. Imaging 2014, 30, 1589-1597. [CrossRef] [PubMed]

14. Schwitter, J.; Nanz, D.; Kneifel, S.; Bertschinger, K.; Buchi, M.; Knusel, P.R.; Marincek, B.; Luscher, T.F.; von Schulthess, G.K. Assessment of myocardial perfusion in coronary artery disease by magnetic resonance: A comparison with positron emission tomography and coronary angiography. Circulation 2001, 103, 2230-2235. [CrossRef]

15. Bernhardt, P.; Levenson, B.; Albrecht, A.; Engels, T.; Strohm, O. Detection of cardiac small vessel disease by adenosine-stress magnetic resonance. Int. J. Cardiol. 2007, 121, 261-266. [CrossRef] [PubMed]

16. Panting, J.R.; Gatehouse, P.D.; Yang, G.Z.; Grothues, F.; Firmin, D.N.; Collins, P.; Pennell, D.J. Abnormal Subendocardial Perfusion in Cardiac Syndrome X Detected by Cardiovascular Magnetic Resonance Imaging. New Engl. J. Med. 2002, 346, 1948-1953. [CrossRef]

17. Mavrogeni, S.; Bratis, K.; Koutsogeorgopoulou, L.; Karabela, G.; Savropoulos, E.; Katsifis, G.; Raftakis, J.; Markousis-Mavrogenis, G.; Kolovou, G. Myocardial perfusion in peripheral Raynaud's phenomenon. Evaluation using stress cardiovascular magnetic resonance. Int. J. Cardiol. 2017, 228, 444-448. [CrossRef]

18. Ishimori, M.L.; Martin, R.; Berman, D.S.; Goykhman, P.; Shaw, L.J.; Shufelt, C.; Slomka, P.J.; Thomson, L.E.; Schapira, J.; Yang, Y.; et al. Myocardial ischemia in the absence of obstructive coronary artery disease in systemic lupus erythematosus. JACC Cardiovasc. Imaging 2011, 4, 27-33. [CrossRef]

19. Greenwood, J.P.; Maredia, N.; Younger, J.F.; Brown, J.M.; Nixon, J.; Everett, C.C.; Bijsterveld, P.; Ridgway, J.P.; Radjenovic, A.; Dickinson, C.J.; et al. Cardiovascular magnetic resonance and single-photon emission computed tomography for diagnosis of coronary heart disease (CE-MARC): A prospective trial. Lancet 2012, 379, 453-460. [CrossRef]

20. Mavrogeni, S.I.; Sfikakis, P.P.; Kitas, G.D.; Kolovou, G.; Tektonidou, M.G. Cardiac involvement in antiphospholipid syndrome: The diagnostic role of noninvasive cardiac imaging. Semin. Arthritis Rheum. 2016, 45, 611-616. [CrossRef]

21. Hochberg, M.C. Updating the American College of Rheumatology revised criteria for the classification of systemic lupus erythematosus. Arthritis Rheum. 1997, 40, 1725. [CrossRef] [PubMed]

22. Schwitter, J.; Wacker, C.M.; Wilke, N.; Al-Saadi, N.; Sauer, E.; Huettle, K.; Schönberg, S.O.; Debl, K.; Strohm, O.; Ahlstrom, H.; et al. Superior diagnostic performance of perfusion-cardiovascular magnetic resonance versus SPECT to detect coronary artery disease: The secondary endpoints of the multicenter multivendor MR-IMPACT II (Magnetic Resonance Imaging for Myocardial Perfusion Assessm. J. Cardiovasc. Magn. Reson. 2012, 14, 61. [CrossRef] [PubMed]

23. Grothues, F.; Moon, J.C.; Bellenger, N.G.; Smith, G.S.; Klein, H.U.; Pennell, D.J. Interstudy reproducibility of right ventricular volumes, function, and mass with cardiovascular magnetic resonance. Am. Heart J. 2004, 147, 218-223. [CrossRef] [PubMed] 
24. Maceira, A.M.; Prasad, S.K.; Khan, M.; Pennell, D.J. Normalized left ventricular systolic and diastolic function by steady state free precession cardiovascular magnetic resonance. J. Cardiovasc. Magn. Reson. 2006, 8, 417-426. [CrossRef] [PubMed]

25. Abdel-Aty, H.; Boyé, P.; Zagrosek, A.; Wassmuth, R.; Kumar, A.; Messroghli, D.; Bock, P.; Dietz, R.; Friedrich, M.G.; Schulz-Menger, J. Diagnostic performance of cardiovascular magnetic resonance in patients with suspected acute myocarditis: Comparison of different approaches. J. Am. Coll. Cardiol. 2005, 45, 1815-1822. [CrossRef] [PubMed]

26. Raman, S.V.; Aneja, A.; Jarjour, W.N. CMR in inflammatory vasculitis. J. Cardiovasc. Magn. Reson. 2012, 14, 82. [CrossRef] [PubMed]

27. Yoon, Y.E.; Kitagawa, K.; Kato, S.; Nakajima, H.; Kurita, T.; Dohi, K.; Ito, M.; Sakuma, H. Prognostic value of unrecognised myocardial infarction detected by late gadolinium-enhanced MRI in diabetic patients with normal global and regional left ventricular systolic function. Eur. Radiol. 2013, 23, 2101-2108. [CrossRef] [PubMed]

28. Pavlik, G.; Bakács, E.; Csajági, E.; Bakács, T.; Noe, J.; Kirschner, R. Improved cardiorespiratory fitness following moderate exercise may encourage inactive people for doable and sustainable behavioral change. J. Sports Med. Phys. Fit. 2019, 59, 502-509. [CrossRef]

29. Giles, J.T.; Malayeri, A.A.; Fernandes, V.; Post, W.; Blumenthal, R.S.; Bluemke, D.; Vogel-Claussen, J.; Szklo, M.; Petri, M.; Gelber, A.C.; et al. Left ventricular structure and function in patients with rheumatoid arthritis, as assessed by cardiac magnetic resonance imaging. Arthritis Rheum. 2010, 62, 940-951. [CrossRef]

30. Espinola-Zavaleta, N.; Alexanderson-Rosas, E.; Granados, N.; Soto, M.; Amigo, M. Myocardial perfusion defects in patients with autoimmune diseases: A prospective study. Analysis of two diagnostic tests. Lupus 2006, 15, 38-43. [CrossRef]

31. Padjas, A.; Płazak, W.; Celińska-Lowenhoff, M.; Mazurek, A.; Perricone, C.; Podolec, P.; Musiał, J. Myocardial Ischaemia, Coronary Atherosclerosis and Pulmonary Pressure Elevation in Antiphospholipid Syndrome Patients. Adv. Clin. Exp. Med. 2016, 25, 1199-1205. [CrossRef] [PubMed]

32. Alexánderson, E.; Gómez-León, A.; Vargas, A.; Romero, J.L.; Sierra Fernandez, C.; Rodriguez Valero, M.; García-Rojas, L.; Meave, A.; Amigo, M.C. Myocardial ischaemia in patients with primary APS: A 13N-ammonia PET assessment. Rheumatology 2008, 47, 894-896. [CrossRef] [PubMed]

33. Kotu, L.P.; Engan, K.; Borhani, R.; Katsaggelos, A.K.; Ørn, S.; Woie, L.; Eftestøl, T. Cardiac magnetic resonance image-based classification of the risk of arrhythmias in post-myocardial infarction patients. Artif. Intell. Med. 2015, 64, 205-215. [CrossRef] [PubMed]

34. Bourantas, C.V.; Nikitin, N.P.; Loh, H.P.; Lukaschuk, E.I.; Sherwi, N.; de Silva, R.; Tweddel, A.C.; Alamgir, M.F.; Wong, K.; Gupta, S.; et al. Prevalence of scarred and dysfunctional myocardium in patients with heart failure of ischaemic origin: A cardiovascular magnetic resonance study. J. Cardiovasc. Magn. Reson. 2011, 13, 53. [CrossRef] [PubMed]

35. Perl, L.; Netzer, A.; Rechavia, E.; Bental, T.; Assali, A.; Codner, P.; Mager, A.; Battler, A.; Kornowski, R.; Lev, E.I. Long-term outcome of patients with antiphospholipid syndrome who undergo percutaneous coronary intervention. Cardiology 2012, 122, 76-82. [CrossRef] [PubMed]

36. Andrade, D.; Tektonidou, M. Emerging Therapies in Antiphospholipid Syndrome. Curr. Rheumatol. Rep. 2016, 18, 22. [CrossRef] [PubMed]

37. Abreu, M.M.; Danowski, A.; Wahl, D.G.; Amigo, M.C.; Tektonidou, M.; Pacheco, M.S.; Fleming, N.; Domingues, V.; Sciascia, S.; Lyra, J.O.; et al. The relevance of "non-criteria" clinical manifestations of antiphospholipid syndrome: 14th International Congress on Antiphospholipid Antibodies Technical Task Force Report on Antiphospholipid Syndrome Clinical Features. Autoimmun. Rev. 2015, 14, 401-414. [CrossRef] [PubMed]

38. Shah, N.R.; Cheezum, M.K.; Veeranna, V.; Horgan, S.J.; Taqueti, V.R.; Murthy, V.L.; Foster, C.; Hainer, J.; Daniels, K.M.; Rivero, J.; et al. Ranolazine in symptomatic diabetic patients without obstructive coronary artery disease: Impact on microvascular and diastolic function. J. Am. Heart Assoc. 2017, 6, e005027. [CrossRef]

39. Tektonidou, M.G.; Andreoli, L.; Limper, M.; Amoura, Z.; Cervera, R.; Costedoat-Chalumeau, N.; Cuadrado, M.J.; Dörner, T.; Ferrer-Oliveras, R.; Hambly, K.; et al. EULAR Recommendations for the management of Antiphospholipid Syndrome in adults. Ann. Rheum. Dis. 2019. [CrossRef] 
40. Tektonidou, M.G.; Andreoli, L.; Limper, M.; Tincani, A.; Ward, M.M. Management of thrombotic and obstetric antiphospholipid syndrome: A systematic literature review informing the EULAR recommendations for the management of antiphospholipid syndrome in adults. RMD Open 2019, 5, e000924. [CrossRef]

41. Winau, L.; Baydes, R.H.; Braner, A.; Drott, U.; Burkhardt, H.; Sangle, S.; D'cruz, D.P.; Carr-White, G.; Marber, M.; Schnoes, K.; et al. High-sensitive troponin is associated with subclinical imaging biosignature of inflammatory cardiovascular involvement in systemic lupus erythematosus. Ann. Rheum. Dis. 2018, 77, 1590-1598. [CrossRef] [PubMed]

(C) 2019 by the authors. Licensee MDPI, Basel, Switzerland. This article is an open access article distributed under the terms and conditions of the Creative Commons Attribution (CC BY) license (http://creativecommons.org/licenses/by/4.0/). 